\title{
Upstream ORFs Influence Translation Efficiency in the Parasite Trypanosoma cruzi
}

\author{
Santiago Radío ${ }^{1,2}$, Beatriz Garat ${ }^{2}$, José Sotelo-Silveira ${ }^{1,3}$ and Pablo Smircich ${ }^{1,2 *}$ \\ 1 Department of Genomics, Instituto de Investigaciones Biológicas Clemente Estable, Ministerio de Educación y Cultura, \\ Montevideo, Uruguay, ${ }^{2}$ Laboratory of Molecular Interactions, Facultad de Ciencias, Universidad de la República, \\ Montevideo, Uruguay, ${ }^{3}$ Department of Cell and Molecular Biology, Facultad de Ciencias, Universidad de la República, \\ Montevideo, Uruguay
}

\section{OPEN ACCESS}

Edited by:

Assaf Katz,

University of Chile, Chile

Reviewed by:

Gary Loughran,

University College Cork, Ireland

Michael James Holmes, Indiana University Bloomington,

United States

*Correspondence:

Pablo Smircich

psmircich@fcien.edu.uy

Specialty section:

This article was submitted to

RNA,

a section of the journal

Frontiers in Genetics

Received: 06 December 2019

Accepted: 12 February 2020

Published: 28 February 2020

Citation:

Radío S, Garat B, Sotelo-Silveira J and Smircich P (2020) Upstream ORFs Influence Translation Efficiency in the Parasite Trypanosoma cruzi.

Front. Genet. 11:166.

doi: 10.3389/fgene.2020.00166
It is generally accepted that the presence of ORFs in the $5^{\prime}$ untranslated region of eukaryotic transcripts modulates the production of proteins by controlling the translation initiation rate of the main CDS. In trypanosomatid parasites, which almost exclusively depend on post-transcriptional mechanisms to regulate gene expression, translation has been identified as a key step. However, the mechanisms of control of translation are not fully understood. In the present work, we have annotated the $5^{\prime} U T R s$ of the Trypanosoma cruzi genome both in epimastigotes and metacyclic trypomastigotes and, using a stringent classification approach, we identified putative regulatory uORFs in about $9 \%$ of the analyzed $5^{\prime}$ UTRs. The translation efficiency (TE) and translational levels of transcripts containing putative repressive uORFs were found to be significantly reduced. These findings are supported by the fact that proteomic methods only identify a low number of proteins coded by transcripts containing repressive UORF. We additionally show that $A U G$ is the main translation initiator codon of repressive UORFs in T. cruzi. Interestingly, the decrease in TE is more pronounced when the UORFs overlaps the main CDS. In conclusion, we show that the presence of the UORF and features such as initiation codon and/or location of the UORFs may be acting to fine tune translation levels in these parasites.

Keywords: Trypanosoma cruzi, uORF, 5'UTR, translation efficiency, translation regulation

\section{INTRODUCTION}

Translation regulation depends on signals that are present mainly at the untranslated regions of the mRNAs (UTRs). Although many regulatory elements are present in the $3^{\prime}$ UTR regions, $5^{\prime}$ UTRs may also contain important $c i s$ acting regulators. In particular, the presence of small open reading frames (upstream ORFs, uORF) in this region has been described as a regulatory mechanism influencing the formation of the translation initiation complex at the initiator codon of the main CDS (McCarthy, 1998). Typically, the presence of uORFs decreases the efficiency of initiation at the main CDS, thus leading to downregulation of its translation rate (Griffin et al., 2001; Vattem and Wek, 2004; Chen et al., 2010). Even though there are cases where the presence of a uORF positively influences the translation efficiency (TE) of the main CDS, these are exceptions (Griffin et al., 2001; Vattem and Wek, 2004; Chen et al., 2010). uORFs may serve as elements that respond to altered environmental conditions, allowing the cells to rapidly adjust their protein production rates (Calvo et al., 2009; Lawless et al., 2009). Features of the uORFs that are efficiently translated 
and negatively regulate the main CDS have been defined. Among them, the uORF position within the 5'UTR, its length, the sequence context of the initiation codon and the overlap with the main CDS have all been shown to affect their regulatory potential (Kozak, 1987, 2002; Rajkowitsch et al., 2004; Wethmar, 2014; Chew et al., 2016; Fervers et al., 2018).

Kinetoplastids are excellent models for the analysis of posttranscriptional mechanisms of gene expression regulation, given that transcription initiation is considered to be constitutive for most of the genome (Soldatos et al., 2010). This implies that the control of the substantial and rapid cell biology changes that take place throughout their life cycle, must rely mostly on post-transcriptional mechanisms (Soldatos et al., 2010). In addition to studies on regulation of mRNA steady state levels and protein degradation or modification, ribosome profiling approaches (Ingolia et al., 2011; Ivanov et al., 2011; Smircich et al., 2015) have confirmed that translation is a key step to regulate gene expression profiles in these organisms (Peabody, 1989; Vasquez et al., 2014; Smircich et al., 2015).

The role of $\mathrm{uORF}$ in kinetoplastids has been studied using reporter genes and through the analysis of Ribo-Seq and proteomic data (Siegel et al., 2005; Jensen et al., 2014; Vasquez et al., 2014; Fervers et al., 2018). Probably due to the use of different definitions, the number of CDS associated to uORFs in the kinetoplastid genomes remains controversial, ranging from 11-22\% in T. brucei (according to (Jensen et al., 2014) or Siegel et al., 2005, respectively) or $29 \%$ in the T. congolense (according to Fervers et al., 2018).

Except for one early report about uORFs composition in four specific genes (Jaeger and Brandao, 2011), a systematic analysis of uORFs in Trypanosoma cruzi is missing. This parasitic protozoan, is the causative agent of the neglected Chagas' disease a neglected infection affecting millions of people in Latin America (Lidani et al., 2019). It has a complex life cycle involving replicative and non-replicative developmental forms both in vertebrate (amastigote and bloodstream trypomastigotes, respectively) and invertebrate hosts (epimastigotes and metacyclic trypomastigotes, respectively) (Tyler and Engman, 2001).

In the present study, we performed a systematic search for uORFs in T. cruzi $5^{\prime}$ UTRs focusing on those with repressive potential. The two life cycle forms of the parasite in the invertebrate host (epimastigotes and metacyclic trypomastigotes) were analyzed to detect putative stage-specific control. Using a stringent approach that considers factors that influence the UORF TE, we found that at least $5 \%$ of the $5^{\prime}$ UTRs of the mRNAs in the T. cruzi genome contain repressive uORFs (approx. $9 \%$ of the analyzed $5^{\prime}$ UTRs). Our results show that genes containing these uORFs have a low translational efficiency and low translation levels in both epimastigotes and metacyclic trypomastigotes insect stages. We also show that the AUG codon is mainly responsible for initiating translation of the uORFs that cause this effect. Additionally, we analyzed the uORFs that overlap the main CDS, a category not previously analyzed in T. cruzi. We found that these overlapping $\mathrm{uORF}$ ( $\mathrm{uORFo}$ ) are associated to the most pronounced decrease of translation efficiency in this organism. In conclusion, we present a repertoire of genes in T. cruzi that exhibit putative repressive uORFs at the $5^{\prime}$ UTR and define characteristics such as the identity of the initiation codon and/or location of the uORFs, that may be contributing to the regulation of gene expression by fine tuning the translation levels.

\section{MATERIALS AND METHODS}

\section{Genomic Data}

The genome sequence of $T$. cruzi strain CL Brener and its gene annotation were obtained from the TriTrypDB database (version 32).

\section{UTRs Determination}

The UTR sequences were determined for the epimastigote and metacyclic trypomastigote stages using the UTRme software (Radio et al., 2018), based on the transcriptomic data (accession numbers ranging from SRR1346053-SRR1346059) data generated in Li et al. (2016). The 5'UTRs with the highest scoring trans-spliced site and longer than 5 nucleotides were used. $82065^{\prime}$ UTR regions were annotated for epimastigotes and 8217 for metacyclic trypomastigotes.

Multigene family members were not analyzed because, due to either the large content of repeated regions or to assembly problems, the $5^{\prime}$ UTR ends are difficult to assess. Besides, their high UTR similarity and abundance would bias the results. Multigene families removed include large families of surface proteins such as MASPs, GP63, Mucins, and TcTS. Finally, we also decided to remove $5^{\prime}$ UTR that contain fragments of T. cruzi protein coding regions, likely produced by assembly or annotation errors. For this purpose, the BLASTX tool (Altschul et al., 1990) was used, eliminating UTRs that returned a hit against the T. cruzi CDS with an $e$-value of less than 0.005 .

\section{uORF Annotation and Classification}

Annotation of $5^{\prime}$ UTR sequences was performed using the UTRme tool (Radio et al., 2018) using data obtained from Li et al. (2016). Open reading frames (ORF) greater than three codons were obtained using the getORF tool (Rice et al., 2000). For all ORFs present in the same strand as the CDS, AUGs in frame to the stop codon of the ORF (uAUG) were searched to define all putative coding sequences. uORFs in which the coding sequences start in the $5^{\prime} \mathrm{UTR}$ region and end within the main CDS (out of frame with respect to the main CDS AUG) were defined as overlapping uORFs (uORFo) while the ones contained entirely in the $5^{\prime} \mathrm{UTR}$ were defined as non-overlapping uORF (uORFno), in this case the frame with respect to the main CDS AUG was not considered.

Putative repressive uORFno elements were filtered considering the following characteristics: (1) the presence of an AUG start codon; (2) a minimum distance of 15 nucleotides from the uAUG to the $5^{\prime}$ end; (3) a maximum distance of 50 nucleotides between the stop codon of the uORF and the AUG of the main CDS; (4) minimum length of 5 amino acids. In the case of the uORFo the requirements were: (1) to start with AUG; (2) a minimum length of three amino acids before the AUG of the main CDS. 
To identify and study uORFs with coding sequences initiating from near cognate codons (NCC), the annotation and classification strategy remained the same with the exception that the initiator codon was changed from AUG to the codon under study.

\section{Translation Efficiency Determination}

Ribosome Profiling data were obtained from the SRA: PRJNA260933 (Smircich et al., 2015) and correspond to the epimastigote and trypomastigote metacyclic stages of T. cruzi. The cutadapt program (Martin, 2011) was used to remove the adapters and filter by quality. The same parameters for the specification of the adapter ( $5^{\prime}$ - CGCCTTGGCCGTACAGCAG - $3^{\prime}$ ), minimum quality allowed (13 phred score), maximum allowed error rate (0.1), and colorspace mode were used for both the RNA-Seq and Ribo-Seq data. As for the length limitation, a size larger than 18 base pairs (bp) and a range between 25 and 40 bp were defined for RNA-Seq and Ribo-Seq data, respectively.

The Bowtie aligner (version 1.2.2) (Langmead et al., 2009) was used to remove contamination produced by readings of ribosomal RNA origin. T. cruzi rRNAs were downloaded from the TriTrypDB database. ShortStack (version 3.6) (Johnson et al., 2016) was used to align the previously obtained reads. The ShortStack program was adapted to accept data from SOLiD technology, while the prediction of secondary structures and micro RNAs was disabled. The mapping mode chosen was the single weighting mode $(\mathrm{U})$ where, only the frequencies of the uniquely aligned reads in the vicinity of the alignment in question are considered in the final weighting. Then, the FeatureCounts module of the SubRead package (v1.5.2) (Liao et al., 2013) was used to quantify the number of reads originated in each transcript or CDS (for RNA-seq or Ribo-seq data, respectively). Finally, the translation efficiency in each stage was obtained with the RiboDiff software (Zhong et al., 2017).

\section{Generation of Sequence Logos}

Sequence logos were generated with the WebLogo 3 online tool, using default parameters (Crooks et al., 2004).

\section{Gene Ontology Enrichment Analysis}

Gene ontology analysis were performed using the tool available for this purpose in the TriTrypDB database for this purpose. The visualization and reduction of the categories was carried out by REVIGO (Supek et al., 2011) in conjunction with the graphic environment of the R language (R Core Team, 2013).

\section{RESULTS AND DISCUSSION}

\section{The T. cruzi Genome Contains Hundreds of uORFs With Repressive Potential}

Precise definition of $5^{\prime}$ UTR sequences was performed using the UTRme tool specifically developed to characterize untranslated regions of trypanosomatid genomes (Radio et al., 2018) using deep transcriptomic data obtained from Li et al. (2016). All open reading frames greater than three codons and present in the same strand as the CDS were then obtained. As described in the methods section, uORFs were classified as overlapping ( $\mathrm{UORFo}$ ) if the coding sequence starts in the $5^{\prime} \mathrm{UTR}$ region and ends within the main CDS (out of frame), or nonoverlapping uORF (uORFno) which are contained entirely in the $5^{\prime}$ UTR of the mRNA.

Since not all uORFs have coding potential nor do they have the same translation initiation efficiency, it is crucial to consider features that influence the repressive capacity of an uORF to define them. According to the literature, putative repressive uORFno elements are more likely to contain the following characteristics: (1) the presence of an AUG start codon (Clements et al., 1988); (2) a minimum distance of 15 nucleotides from the 5 ' end, since shorter distances render difficult the assembly of the translation machinery at the uORF initiation codon (Vilela and McCarthy, 2003); (3) a maximum distance of 50 nucleotides between the stop codon of the UORF and the AUG of the main CDS, as shorter re-initiation times are associated to greater repressive potential (Chew et al., 2016) and (4) minimum length of 5 amino acids, as the longer the uORF length, the lower the probability of translation reinitiating at the main CDS (Kozak, 2001; Rajkowitsch et al., 2004). In the case of the uORFo, in which the coding sequences ends within the main CDS the requirements were: (1) to start with AUG; and (2) at least a minimum length of three amino acids before the AUG of the main CDS. Both types of uORFs passing the requirements were classified as repressive uORFs and $5^{\prime} \mathrm{UTR}$ regions that have one or more putative repressive elements were also classified as repressive. In turn, 5'UTRs that do not contain repressive elements, have a size greater than 50 nucleotides and do not contain AUGs were defined as non-repressive. Regions that do not fulfill any of the above categories were not assigned any classification.

After performing the above classification, the $5^{\prime} \mathrm{UTR}$ regions of T. cruzi mRNAs in both insect stages were studied. For the epimastigote stage, 6744 regions were analyzed, 568 of which (8.4\%) classified as repressive. Among them, 111 regions contain only uORFo and 160 contain only uORFno, while the remaining regions (297) encompass elements of both categories. In addition, 3375 regions (50\%) were classified as non-repressive. In the trypomastigote stage, 6750 mRNAs were analyzed, and a similar distribution was observed, 602 regions (8.9\%) being classified as repressive. Among them, 231 carry both repressive types of uORFs, while 204 contain only uORFo and 167 only uORFno. Finally, 3333 regions (49\%) were classified as non-repressive. These numbers are summarized in Table 1 and the list of gene identifiers for the analyzed UTRs can be found in Supplementary Table 1. Interestingly, the percentage of genes that have putative repressive elements is similar to that detected in T. brucei (11\%) by Jensen et al. (2014). The observed differences with other reports (Vasquez et al., 2014; Fervers et al., 2018) are most likely due to the different inclusion criteria applied in each work.

\section{mRNAs Containing Putative Repressive uORFs Are Characterized by a Low TE in}

\section{T. cruzi}

An open reading frame with repressive potential in the $5^{\prime} \mathrm{UTR}$ region of a gene could decrease its translation efficiency. To independently assess the repressive effect of the two defined 
TABLE 1 | Number of genes presenting UORFs in T. cruzi epimastigotes and metacyclic trypomastigote stages.

\begin{tabular}{lccccc}
\hline $\begin{array}{l}\text { T. cruzi life cycle } \\
\text { stage }\end{array}$ & \multicolumn{3}{c}{ Repressive uORFs } & $\begin{array}{c}\text { Non- } \\
\text { repressive }\end{array}$ & $\begin{array}{c}\text { Total } \\
\text { analyzed }\end{array}$ \\
\cline { 2 - 4 } & uORFo & uORFno & Both & & \\
\hline Epimastigote & 111 & 160 & 297 & 3375 & 6744 \\
Trypomastigote & 204 & 167 & 231 & 3333 & 6750 \\
\hline
\end{tabular}

The number of repressive (either overlapping, non-overlapping or both) and nonrepressive UORFS are indicated. Also, the total number of transcripts analyzed for each T. cruzi stage is shown.

categories, the TE values of the genes whose 5'UTR regions contain only repressive uORFno or only uORFo were calculated using Ribodiff and available ribosome profiling data obtained by our group (Smircich et al., 2015). TE values were also obtained for the non-repressive category and for all the genes. Translation efficiency values were then correlated to the presence of repressive uORFs in the $5^{\prime}$ UTRs. The results show that the TE is indeed significantly correlated with the presence of uORFs that have repressive potential and with the subcategory to which they belong (Figure 1A).

Interestingly, genes containing only uORFo have the lowest TE. As ribosomes translating an uORFo are out of frame, they will read through the main CDS AUG, thus establishing an important translational control mechanism. The effect of naturally occurring uORFo has been poorly described in the literature, there are only a few previous reports on it (Wethmar, 2014). Recently, Fervers et al. observed that in T. congolense, as the distance between the UAUG and the AUG of the main CDS decreases, the translation efficiency does too, reaching the maximum decrease when the overlap occurs (Fervers et al., 2018), suggesting that this effect may be shared among trypanosomatids.

In the case of genes containing only uORFno, the drop in observed TE is lower than for those containing only uORFo. However, a clear repressive effect is seen, particularly when compared with the TE of the transcripts with $5^{\prime}$ UTRs categorized as non-repressive. The TE of this later group is the highest in the entire comparison, suggesting that they indeed lack repressive uORFs and highlighting the relevance of these elements in translation control. In turn, the TE of all the $5^{\prime} \mathrm{UTR}$ regions shows an intermediate value between the repressive and non-repressive categories as expected considering the latter results.

In the metacyclic trypomastigote stage, a similar situation is evident even though the data show a higher dispersion, specially for the uORFno-containing genes (Figure 1B). It is worth noting that in the metacyclic trypomastigote stage, genome-wide translational repression has been described (Smircich et al., 2015), implying the existence of other regulatory mechanisms that exert a more significant effect on translation control.

It has been reported that the higher the number of uORFs in the 5'UTR of an mRNA, the higher the decrease in its translation efficiency (Chew et al., 2016). However, no correlation between the number of $\mathrm{UORF}$ present in the $5^{\prime} \mathrm{UTR}$ region and the translation efficiency of the genes was found in our model (Supplementary Figure 1).
Globally, the results presented here are in good agreement with those previously obtained in other trypanosomatids, suggesting that the presence of open reading frames with regulatory potential in the $5^{\prime} \mathrm{UTR}$ regions is a contributing factor to translational control in these organisms. We also demonstrate that overlapping upstream open reading frames achieve the highest level of repression in T. cruzi. Additionally, the features we selected for uORF classification were confirmed to behave as good indicators of the repressive potential of uORFs, both in the case of repressive and non-repressive categories. Finally, we produced a dataset of T. cruzi genes where uORFs are likely acting as important regulatory elements.

\section{AUG Is the Main Initiation Codon of the Repressive uORF in T. cruzi}

Pioneering studies initially proposed that uORFs with non-AUG initiation codons have poor translation efficiency (Clements et al., 1988). Later, several reports claimed that the majority of the translational initiation codons for uORFs were non-canonical (not AUG) (Ingolia et al., 2011; Fritsch et al., 2012; Lee et al., 2012). More recently, through the analysis of Ribo-seq data, it has been suggested that the translation efficiency of non-AUG initiating uORFs is low (Michel et al., 2014).

This led us to ask whether the presence of AUG as a start codon of an uORF can modulate the repressive effect on translation efficiency in T. cruzi. To find an answer to this question, we evaluated if uORFno initiating from each of the 61 codons would also cause a general effect on TE of the main CDS, as observed for AUG. Putative repressive uORFs were defined by maintaining the requirements to classify uORFs in this category (length, distance to the AUG, distance to the $5^{\prime}$ end) and only changing the criteria for the identity of the initiation codon. Thus, for each codon a set of uORFno was determined while preserving the rest of the requirements as before. Then, each new set of uORFs was correlated with the TE distribution of the genes, as done for before AUG initiating uORFs.

In the epimastigote stage, the translation efficiency of genes with uORFno using AUG as initiation codon is significantly lower (non-parametric Mann-Whitney $U$ test $<0.01$ ) compared to any other codon (Figure 2A and Supplementary Figure 2). Our results show no evidence of any particular behavior of mRNAs containing uORFs beginning with near cognate codons (NCC) known to be capable of translation initiation in other systems (CUG, UUG, GUG, ACG, AUA, and AUU) (Peabody, 1989; Ivanov et al., 2011). A similar situation is observed for the metacyclic trypomastigote stage (Figure 2B), the uORFno starting with AUG also being the only initiation codon associated with statistical significance to a low TE.

This approach allowed us to determine that AUG is the main initiation codon that generates repressive uORFno in T. cruzi. This finding further supports our original criteria. It is worth noting that this analysis does not eliminate the possibility that NCC initiate translation in specific uORFs with important regulatory consequences for the affected CDS. Indeed, experimentally assessing the translation of uORFs initiated by NCC would provide interesting insights in this regard. Even 

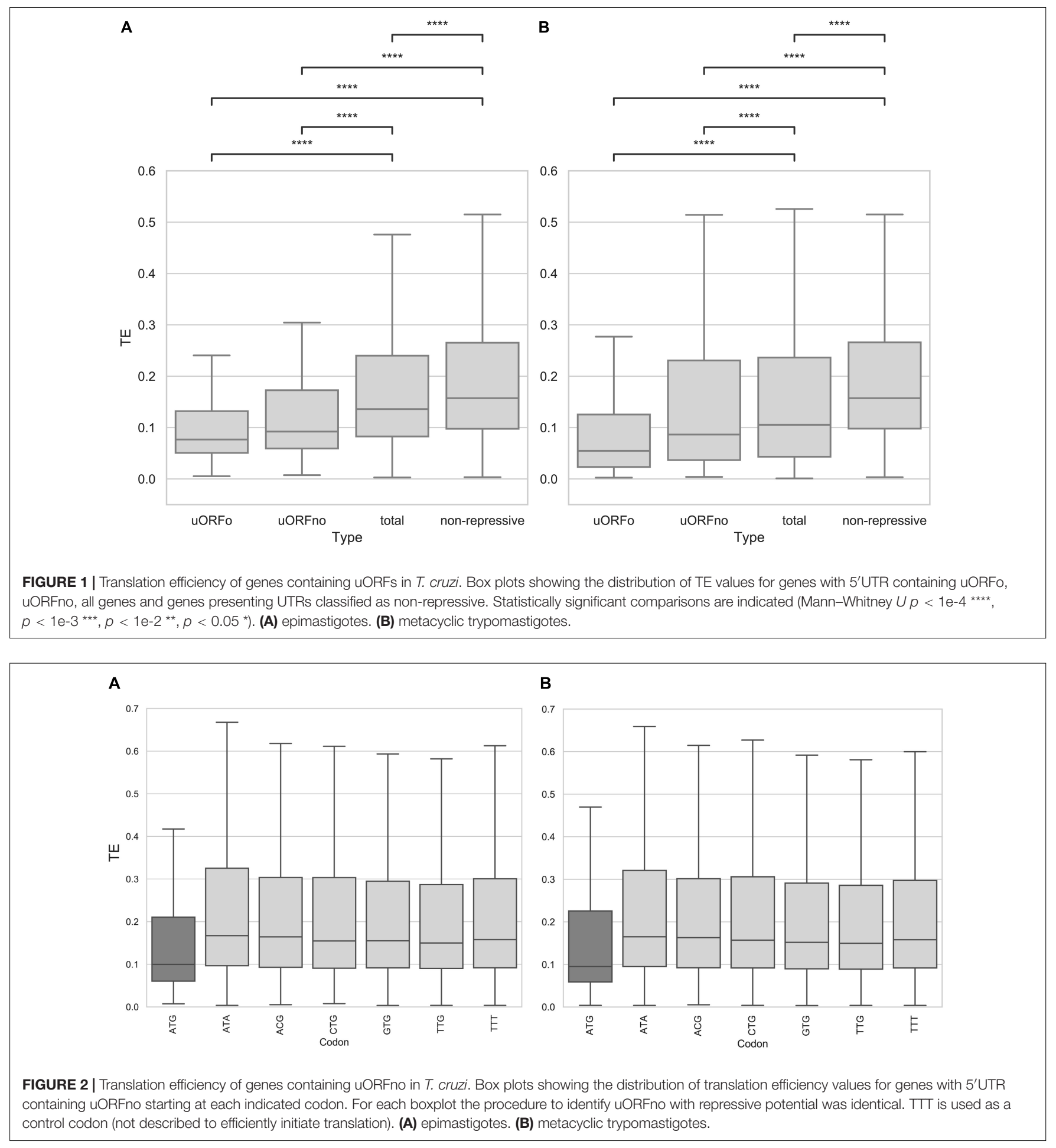

though Ribo-seq data has been used to this end in other models, the sensitivity reached by our data for the $5^{\prime}$ UTR regions did not allow us to address this issue.

Kozak proposed that translation efficiency is strongly determined by the context of the initiating AUG (Kozak, 1978, 2002). In many organisms including T. brucei and L. major, only some of the characteristics defined by Kozak for mammalian cells are preserved, mainly the presence of $\mathrm{A}$ in the -3 position (Nakagawa et al., 2008). To further address this question in T. cruzi, we studied the primary sequence context of the initiator AUG of all main CDSs and repressive uORFno in the epimastigote stage (10 nt flanking the $\mathrm{A}$ in position +1 of the CDS). While the overrepresentation of $A$ at position -3 is not as evident as for the other trypanosomatids, a clear purine 


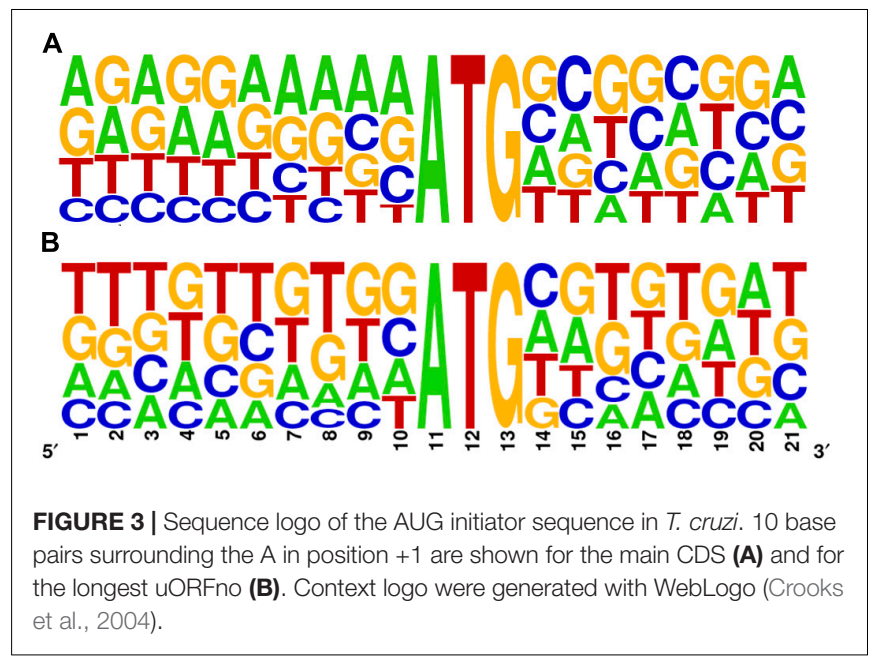

enrichment is found for the main CDS AUG. However, this is not observed for the uORFs AUG (Figure 3), suggesting that initiation driven from the $\mathrm{uAUG}$ is not as efficient as from the main CDS AUG.

\section{The Presence of uORFno Is Correlated to the 5'UTR Length}

As the presence of a uORFno will set a minimum length for the $5^{\prime} \mathrm{UTR}$, we explored the size of this region. We found that the $5^{\prime}$ UTR size of the uORFno associated genes is significantly larger (Mann-Whitney $U$ test, $p$-value $<0.001$ ) than the rest of the groups, for both the epimastigotes or metacyclic trypomastigote stages, as can be seen in Figure 4. Interestingly, the median size of repressive UTRs containing uORFno almost triples the length of the average T. cruzi $5^{\prime} \mathrm{UTR}$. This finding implies that either the maintenance of uORFno increases the size of these regions, or that the length of the $5^{\prime}$ UTR by itself is a determinant of the repressive potential. The latter is not a likely explanation as there is no general genome wide correlation between $5^{\prime}$ UTR length and TE (Supplementary Figure 3).

In addition, this observation suggests that the minimum length of the $5^{\prime}$ UTRs of genes regulated by uORFno might be evolutionary restricted to allow for the presence of the regulatory element. In support of this hypothesis, regions containing uORFo (elements that repress TE through a size independent mechanism), have the shortest $5^{\prime}$ UTRs of all the groups.

\section{Genes Associated to Repressive 5'UTRs Have Low Expression in T. cruzi}

In order to study the association between the presence of repressive $\mathrm{uORF}$ and the translational level of the CDS, we comparatively analyzed the translation rates of genes containing repressive and non-repressive uORFs using our RiboSeq data. We found that genes with repressive uORFs have a decreased the number of ribosomal footprints compared to genes with non-repressive UTRs. Accordingly, the latter
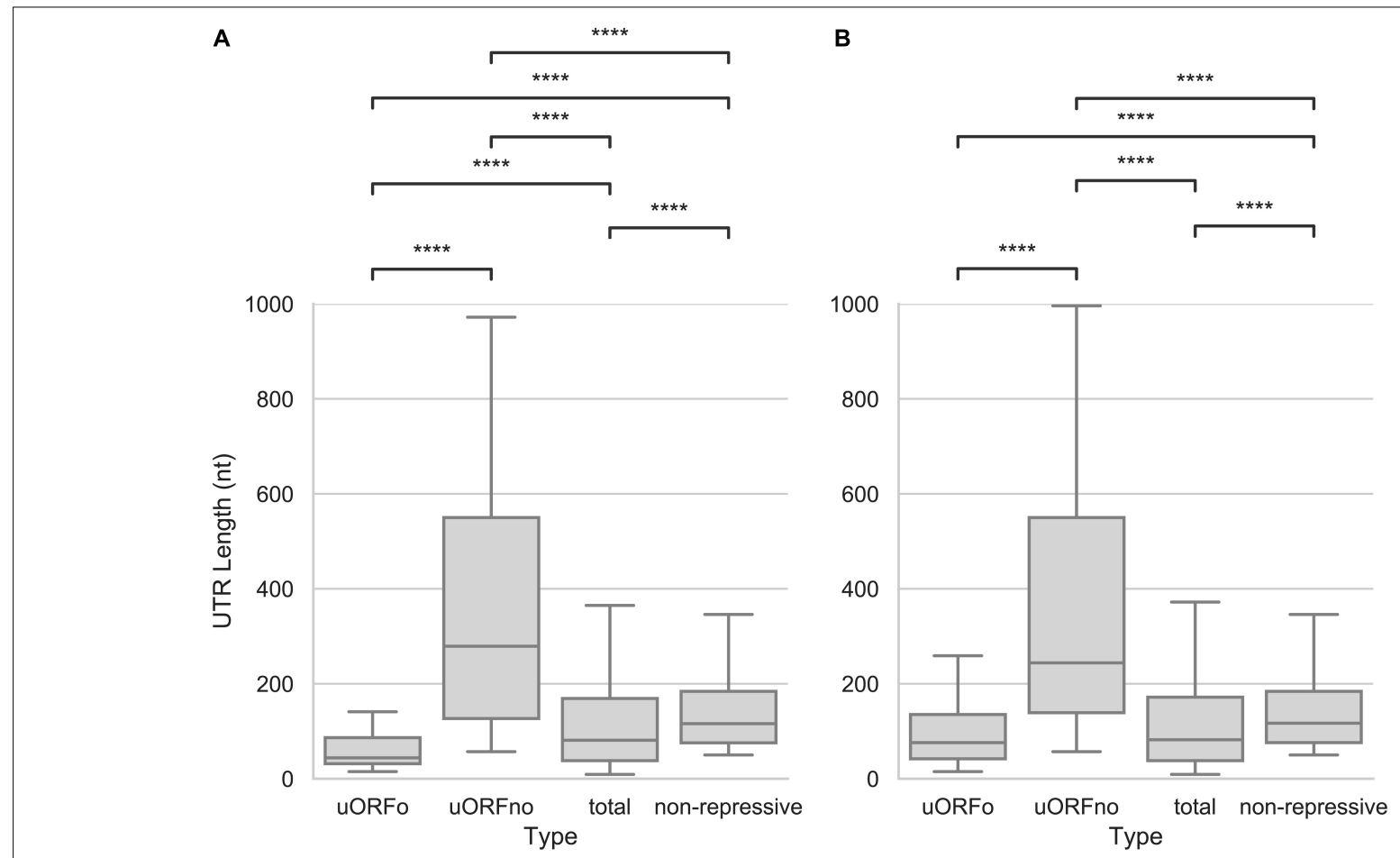

FIGURE 4 | Length of the $5^{\prime}$ UTRs in T. cruzi. Box plots showing the distribution of $5^{\prime}$ UTR lengths for genes with $5^{\prime}$ UTR containing uORFo, uORFno, all genes and genes presenting UTRs classified as non-repressive. Statistically significant comparisons are indicated (Mann-Whitney $U p<1 \mathrm{e}-4^{\star \star \star \star}, p<1 \mathrm{e}-3^{\star \star \star}, p<1 \mathrm{e}-2^{\star \star}$, $p<0.05$ *). (A) epimastigotes. (B) metacyclic trypomastigotes. 
are also have the greatest translation rates (Figure 5A and Supplementary Figure 4A).

This effect is also reflected in the available proteomic data (de Godoy et al., 2012) where most proteins translated from genes containing repressive uORFs are not detected. Indeed, we found a significant underrepresentation of these genes in proteomic databases and an overrepresentation of proteins whose mRNAs do not contain repressive uORFs (Thermo Fisher Scientific test $<0.05$, Figure 5B and Supplementary Figure 4B). As detection in proteomic studies is biased by the relative amount of protein in the sample, these data suggest low levels for proteins coded by repressive uORF containing mRNAs.

Furthermore, genes with non-repressive 5'UTRs show ontology term enrichment with a trend for housekeeping functions such as catabolic processes, cell movement and transport, which are generally associated with high expression protein levels. Conversely, genes with $5^{\prime}$ UTR containing repressive uORFs show enrichment in translational elongation
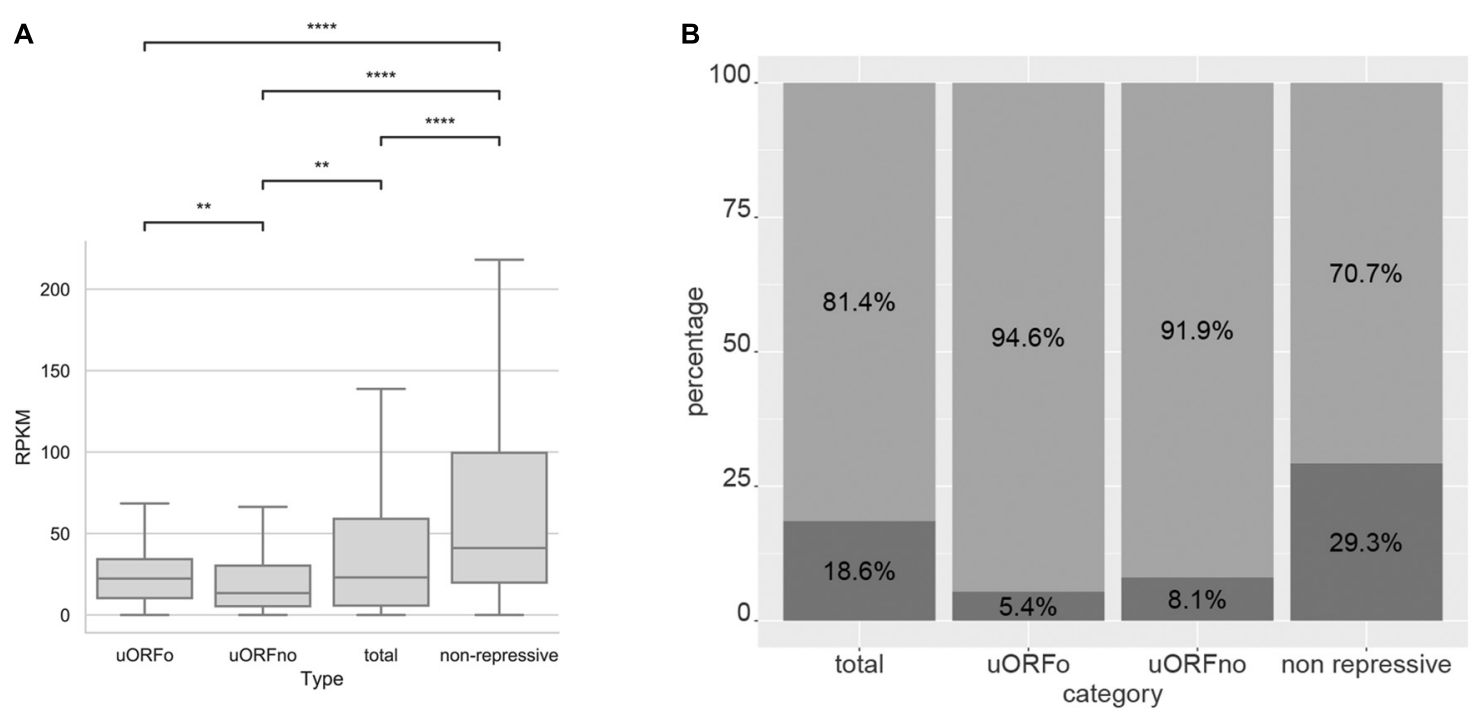

FIGURE 5 | Analysis of the translation of genes with UORFs in T. cruzi epimastigotes. (A) Ribosomal footprints obtained from Smircich et al. (2015) were analyzed for genes with 5'UTR containing uORFo, uORFno, all genes and genes presenting UTRs classified as non-repressive. Boxplot of ribosomal footprints (RPKM) for genes in each category. Statistically significant comparisons are indicated (Mann-Whitney $U p<1 \mathrm{e}-4^{\star \star \star \star}, p<1 \mathrm{e}-3^{* \star *}, p<1 \mathrm{e}-2^{* \star}, p<0.05^{*}$ ). (B) Fraction of the genes belonging to each category present in the proteomic data of de Godoy et al. (2012) are represented in dark gray. A Fisher's exact test was used to assess over or down representation of the number of detected proteins in proteomic experiments for each category compared to the number detected in the total proteome (all comparisons are significant $p<0.05)$.
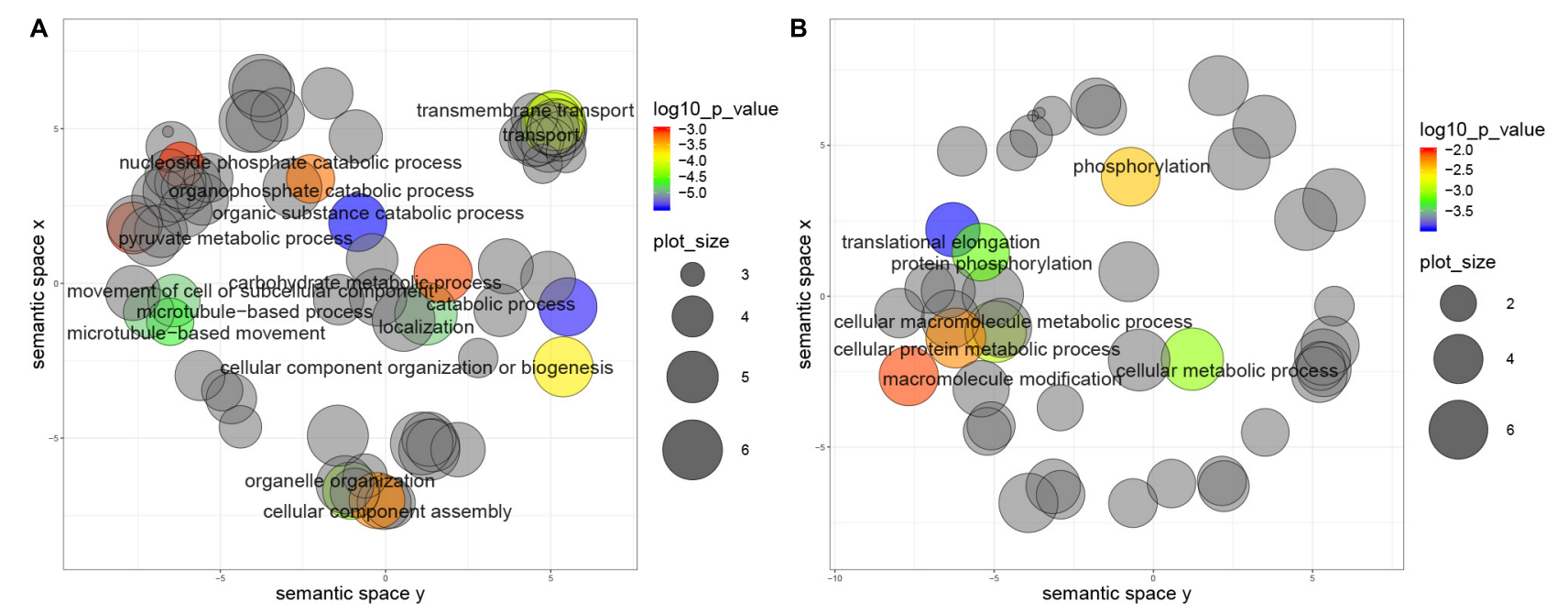

FIGURE 6 | Biological process categories overrepresented in genes with uORFs in T. cruzi epimastigotes. Grouping reveals functionally related categories, size shows the frequency of the UniProtKB database category and color indicates a $p$ value $<0.01$. (A) Genes with non-repressive $5^{\prime} \cup T R s$, (B) Genes with repressive 5'UTRs. 
and phosphorylation terms (Figures 6A,B). Similar results were obtained for the metacyclic trypomastigote stage, so we can conclude that this is not a stage specific characteristic (data not shown).

Finally, to evaluate if alternative $5^{\prime}$ UTR processing could provide a mechanism to regulate the presence of uORFs (and thus the TE) between T. cruzi life stages, we searched for differential $5^{\prime}$ UTR processing between epimatigotes and metacyclic trypomastigotes. First, we observed that most genes either share their main 5'UTR splice site (83\% of the 3245 analyzed), or have sites are less than $10 \mathrm{nt}$ apart (60\% of the nonidentical ones) (Supplementary Figure 5). This indicates that the presence or absence of uORFs is not a general mechanism to regulate differential TE between these life-cycle stages. We cannot discard that differential translation efficiency of the uORFs themselves might provide a regulatory mechanism of the main CDS. This requires further investigation as it cannot be assessed with our current data.

\section{FINAL REMARKS}

Overall, the results here presented allow us to conclude that uORFs are a frequent mechanism to fine tune translation efficiency in T. cruzi. Characteristics intrinsic to the uORF - such as its position within the $5^{\prime} \mathrm{UTR}$, size and start codon - influence their ability to exert this effect. Genes with repressive uORFs have low levels of ribosomal footprints and are underrepresented in proteomic data, while the opposite is observed for genes with $5^{\prime}$ UTR regions classified as non-repressive. Finally, differential $5^{\prime}$ UTR processing does not seem to be a general mechanism to regulate their TE between the parasite life-cycle stages analyzed.

\section{REFERENCES}

Altschul, S. F., Gish, W., Miller, W., Myers, E. W., and Lipman, D. J. (1990). Basic local alignment search tool. J. Mol. Biol. 215, 403-410. doi: 10.1016/S00222836(05)80360-2

Calvo, S. E., Pagliarini, D. J., and Mootha, V. K. (2009). Upstream open reading frames cause widespread reduction of protein expression and are polymorphic among humans. Proc. Natl. Acad. Sci. U.S.A. 106, 7507-7512. doi: 10.1073/pnas. 0810916106

Chen, Y. J., Tan, B. C., Cheng, Y. Y., Chen, J. S., and Lee, S. C. (2010). Differential regulation of CHOP translation by phosphorylated eIF4E under stress conditions. Nucleic Acids Res. 38, 764-777. doi: 10.1093/nar/gkp1034

Chew, G. L., Pauli, A., and Schier, A. F. (2016). Conservation of uORF repressiveness and sequence features in mouse, human and zebrafish. Nat. Commun. 7:11663. doi: 10.1038/ncomms 11663

Clements, J. M., Laz, T. M., and Sherman, F. (1988). Efficiency of translation initiation by non-AUG codons in Saccharomyces cerevisiae. Mol. Cell Biol. 8, 4533-4536. doi: $10.1128 / \mathrm{mcb} .8 .10 .4533$

Crooks, G. E., Hon, G., Chandonia, J. M., and Brenner, S. E. (2004). WebLogo: a sequence logo generator. Genome Res. 14, 1188-1190. doi: 10.1101/gr.849004

de Godoy, L. M., Marchini, F. K., Pavoni, D. P., Rampazzo Rde, C., Probst, C. M., Goldenberg, S., et al. (2012). Quantitative proteomics of Trypanosoma cruzi during metacyclogenesis. Proteomics 12, 2694-2703. doi: 10.1002/pmic. 201200078

Fervers, P., Fervers, F., Makalowski, W., and Jakalski, M. (2018). Life cycle adapted upstream open reading frames (uORFs) in Trypanosoma congolense: a posttranscriptional approach to accurate gene regulation. PLoS One 13:e0201461. doi: 10.1371/journal.pone.0201461

\section{DATA AVAILABILITY STATEMENT}

All datasets generated for this study are included in the article/Supplementary Material.

\section{AUTHOR CONTRIBUTIONS}

SR and PS designed the methodology. SR performed the analysis. JS-S, BG, and PS acquired the financial support. PS coordinated the project. All authors wrote and reviewed the manuscript.

\section{FUNDING}

This project was supported by the ANII, FCE_3_2016_1_126317; CSIC, I+D research groups program 108725. SR received scholarships from the ANII. SR and PS received financial support from the PEDECIBA.

\section{ACKNOWLEDGMENTS}

The authors would like to acknowledge Dr. Martin Ciganda for careful revision of the manuscript.

\section{SUPPLEMENTARY MATERIAL}

The Supplementary Material for this article can be found online at: https://www.frontiersin.org/articles/10.3389/fgene. 2020.00166/full\#supplementary-material

Fritsch, C., Herrmann, A., Nothnagel, M., Szafranski, K., Huse, K., Schumann, F., et al. (2012). Genome-wide search for novel human uORFs and N-terminal protein extensions using ribosomal footprinting. Genome Res. 22, 2208-2218. doi: $10.1101 /$ gr.139568.112

Griffin, E., Re, A., Hamel, N., Fu, C., Bush, H., McCaffrey, T., et al. (2001). A link between diabetes and atherosclerosis: glucose regulates expression of CD36 at the level of translation. Nat. Med. 7, 840-846. doi: 10.1038/89969

Ingolia, N. T., Lareau, L. F., and Weissman, J. S. (2011). Ribosome profiling of mouse embryonic stem cells reveals the complexity and dynamics of mammalian proteomes. Cell 147, 789-802. doi: 10.1016/j.cell.2011.10.002

Ivanov, I. P., Firth, A. E., Michel, A. M., Atkins, J. F., and Baranov, P. V. (2011). Identification of evolutionarily conserved non-AUG-initiated N-terminal extensions in human coding sequences. Nucleic Acids Res. 39, 4220-4234. doi: 10.1093/nar/gkr007

Jaeger, L. H., and Brandao, A. (2011). The composition of upstream open reading frames (uORF) in four genes from Trypanosoma cruzi typical strains. Parasitol. Res. 109, 1205-1208. doi: 10.1007/s00436-011-2430-4

Jensen, B. C., Ramasamy, G., Vasconcelos, E. J., Ingolia, N. T., Myler, P. J., and Parsons, M. (2014). Extensive stage-regulation of translation revealed by ribosome profiling of Trypanosoma brucei. BMC Genomics 15:911. doi: 10.1186/ 1471-2164-15-911

Johnson, N. R., Yeoh, J. M., Coruh, C., and Axtell, M. J. (2016). Improved placement of multi-mapping small RNAs. G3 6, 2103-2111. doi: 10.1534/g3. 116.030452

Kozak, M. (1978). How do eucaryotic ribosomes select initiation regions in messenger RNA? Cell 15, 1109-1123. doi: 10.1016/0092-8674(78)90039-9

Kozak, M. (1987). Effects of intercistronic length on the efficiency of reinitiation by eucaryotic ribosomes. Mol. Cell Biol. 7, 3438-3445. doi: 10.1128/mcb.7.10.3438 
Kozak, M. (2001). Constraints on reinitiation of translation in mammals. Nucleic Acids Res. 29, 5226-5232. doi: 10.1093/nar/29.24.5226

Kozak, M. (2002). Pushing the limits of the scanning mechanism for initiation of translation. Gene 299, 1-34. doi: 10.1016/s0378-1119(02)01056-9

Langmead, B., Trapnell, C., Pop, M., and Salzberg, S. L. (2009). Ultrafast and memory-efficient alignment of short DNA sequences to the human genome. Genome Biol. 10:R25. doi: 10.1186/gb-2009-10-3-r25

Lawless, C., Pearson, R. D., Selley, J. N., Smirnova, J. B., Grant, C. M., Ashe, M. P., et al. (2009). Upstream sequence elements direct post-transcriptional regulation of gene expression under stress conditions in yeast. BMC Genomics 10:7. doi: 10.1186/1471-2164-10-7

Lee, S., Liu, B., Lee, S., Huang, S. X., Shen, B., and Qian, S. B. (2012). Global mapping of translation initiation sites in mammalian cells at single-nucleotide resolution. Proc. Natl. Acad. Sci. U.S.A. 109, E2424-E2432. doi: 10.1073/pnas. 1207846109

Li, Y., Shah-Simpson, S., Okrah, K., Belew, A. T., Choi, J., Caradonna, K. L., et al. (2016). Transcriptome remodeling in Trypanosoma cruzi and human cells during intracellular infection. PLoS Pathog. 12:e1005511. doi: 10.1371/journal. ppat. 1005511

Liao, Y., Smyth, G. K., and Shi, W. (2013). The Subread aligner: fast, accurate and scalable read mapping by seed-and-vote. Nucleic Acids Res. 41:e108. doi: $10.1093 /$ nar/gkt214

Lidani, K. C. F., Andrade, F. A., Bavia, L., Damasceno, F. S., Beltrame, M. H., Messias-Reason, I. J., et al. (2019). Chagas disease: from discovery to a worldwide health problem. Front. Public Health 7:166. doi: 10.3389/fpubh.2019. 00166

Martin, M. (2011). Cutadapt removes adapter sequences from high-throughput sequencing reads. EMBnet. J. 17, 10-12.

McCarthy, J. E. (1998). Posttranscriptional control of gene expression in yeast. Microbiol. Mol. Biol. Rev. 62, 1492-1553. doi: 10.1128/mmbr.62.4.1492-1553. 1998

Michel, A. M., Andreev, D. E., and Baranov, P. V. (2014). Computational approach for calculating the probability of eukaryotic translation initiation from riboseq data that takes into account leaky scanning. BMC Bioinformatics 15:380. doi: 10.1186/s12859-014-0380-4

Nakagawa, S., Niimura, Y., Gojobori, T., Tanaka, H., and Miura, K. (2008). Diversity of preferred nucleotide sequences around the translation initiation codon in eukaryote genomes. Nucleic Acids Res. 36, 861-871. doi: 10.1093/nar/ gkm1102

Peabody, D. S. (1989). Translation initiation at non-AUG triplets in mammalian cells. J. Biol. Chem. 264, 5031-5035.

R Core Team, (2013). R: A Language and Environment for Statistical Computing. Vienna: R Core Team.

Radio, S., Fort, R. S., Garat, B., Sotelo-Silveira, J., and Smircich, P. (2018). UTRme: a scoring-based tool to annotate untranslated regions in trypanosomatid genomes. Front. Genet. 9:671. doi: 10.3389/fgene.2018.00671

Rajkowitsch, L., Vilela, C., Berthelot, K., Ramirez, C. V., and McCarthy, J. E. (2004). Reinitiation and recycling are distinct processes occurring downstream of translation termination in yeast. J. Mol. Biol. 335, 71-85. doi: 10.1016/j.jmb. 2003.10.049

Rice, P., Longden, I., and Bleasby, A. (2000). EMBOSS: the european molecular biology open software suite. Trends Genet. 16, 276-277. doi: 10.1016/s01689525(00)02024-2

Siegel, T. N., Tan, K. S., and Cross, G. A. (2005). Systematic study of sequence motifs for RNA trans splicing in Trypanosoma brucei. Mol. Cell Biol. 25, 9586-9594. doi: 10.1128/MCB.25.21.9586-9594.2005

Smircich, P., Eastman, G., Bispo, S., Duhagon, M. A., Guerra-Slompo, E. P., Garat, B., et al. (2015). Ribosome profiling reveals translation control as a key mechanism generating differential gene expression in Trypanosoma cruzi. BMC Genomics 16:443. doi: 10.1186/s12864-015-1563-8

Soldatos, T. G., O’Donoghue, S. I., Satagopam, V. P., Jensen, L. J., Brown, N. P., Barbosa-Silva, A., et al. (2010). Martini: using literature keywords to compare gene sets. Nucleic Acids Res. 38, 26-38. doi: 10.1093/nar/gkp876

Supek, F., Bosnjak, M., Skunca, N., and Smuc, T. (2011). REVIGO summarizes and visualizes long lists of gene ontology terms. PLoS One 6:e21800. doi: 10.1371/ journal.pone.0021800

Tyler, K. M., and Engman, D. M. (2001). The life cycle of Trypanosoma cruzi revisited. Int. J. Parasitol. 31, 472-481. doi: 10.1016/s0020-7519(01)00153-9

Vasquez, J. J., Hon, C. C., Vanselow, J. T., Schlosser, A., and Siegel, T. N. (2014). Comparative ribosome profiling reveals extensive translational complexity in different Trypanosoma brucei life cycle stages. Nucleic Acids Res. 42, 3623-3637. doi: 10.1093/nar/gkt1386

Vattem, K. M., and Wek, R. C. (2004). Reinitiation involving upstream ORFs regulates ATF4 mRNA translation in mammalian cells. Proc. Natl. Acad. Sci. U.S.A. 101, 11269-11274. doi: 10.1073/pnas.0400541101

Vilela, C., and McCarthy, J. E. (2003). Regulation of fungal gene expression via short open reading frames in the mRNA 5 ' untranslated region. Mol. Microbiol. 49, 859-867. doi: 10.1046/j.1365-2958.2003.03622.x

Wethmar, K. (2014). The regulatory potential of upstream open reading frames in eukaryotic gene expression. Wiley Interdiscip. Rev. RNA 5, 765-778. doi: 10.1002/wrna.1245

Zhong, Y., Karaletsos, T., Drewe, P., Sreedharan, V. T., Kuo, D., Singh, K., et al. (2017). RiboDiff: detecting changes of mRNA translation efficiency from ribosome footprints. Bioinformatics 33, 139-141. doi: 10.1093/bioinformatics/ btw585

Conflict of Interest: The authors declare that the research was conducted in the absence of any commercial or financial relationships that could be construed as a potential conflict of interest.

Copyright (C) 2020 Radio, Garat, Sotelo-Silveira and Smircich. This is an open-access article distributed under the terms of the Creative Commons Attribution License (CC BY). The use, distribution or reproduction in other forums is permitted, provided the original author(s) and the copyright owner(s) are credited and that the original publication in this journal is cited, in accordance with accepted academic practice. No use, distribution or reproduction is permitted which does not comply with these terms. 